\title{
SUBMANIFOLDS WITH PARALLEL RICCI TENSOR
}

\author{
By Yoshio MatsuYAMA
}

Let $\tilde{M}^{n+p}(\tilde{c})$ be a Riemannian $(n+p)$-manifold of constant sectional curvature $\tilde{c}$, which is called a real space form. If $\tilde{c}=0$, then $\tilde{M}^{n+p}(0)$ denotes the Euclidean $(n+p)$-space $R^{n+p}$. If $\tilde{c}>0$ (resp. $\tilde{c}<0$ ), then $\tilde{M}^{n+p}(\tilde{c})$ denotes the Euclidean $(n+p)$-sphere $S^{n+p}(\tilde{c})$ (resp. the hyperbolic $(n+p)$-space $\left.H^{n+p}(\tilde{c})\right)$ in $R^{n+p+1}$. We consider submanifolds isometrically immersed in a real space form. Ryan [5] showed: Let $M^{n}(n>2)$ be a hypersurface in $\tilde{M}^{n+1}(\tilde{c})$. If $M$ is not of constant curvature $\tilde{c}$ and if the Ricci tensor of $M$ is parallel, then either $M$ is locally isometric to the product $M_{1}^{k} \times M_{2}^{n-k}, 0 \leqq k \leqq n$ (if $\tilde{c}=0$, then $k \neq 2$ ), or $\tilde{c}=0$ and the rank of the second fundamental form $A\left(=A_{1}\right)$ is equal to 2 everywhere. Here, $M_{1}^{k}$ is a sphere of some radius contained in some Euclidean space $R^{k+1}$ (resp. $M_{2}^{n-k}$ is one in some Euclidean space perpendicular to $R^{k+1}$ ), except possibly one of $M_{i}(i=1,2)$ is a Euclidean space (this can only occur if $\tilde{c} \leqq 0$, and $k=0$ or $n$ if $\tilde{c}<0$ ) or a hyperbolic space with some negative curvature $\bar{c}$ (this can only occur if $\tilde{c}<0$ ). In order to prove the above result Ryan made use of the following remarkable result ([5]): let $M^{n}$ be as above. If the mean curvature is constant, then the second fundamental form of $M$ is parallel.

On the other hand, in [3], [4] the author proved:

THEOREM. Let $M^{n}$ be an $n(>2)$-dimensional minimal Einstein submanifold in an $(n+2)$-dimensional space form $\tilde{M}^{n+2}(\tilde{c})$ with constant curvature $\tilde{c}$. Then the second fundamental form of $M$ is parallel and that (1) if $\tilde{c} \leqq 0$, then $M$ is totally geodesic and that (2) if $\tilde{c}>0$, then either $M$ is totally geodesic or locally isometric to the product $S^{m}\left(\frac{1}{\sqrt{ } 2 \tilde{c}}\right) \times S^{m}\left(\frac{1}{\sqrt{ } 2 \tilde{c}}\right)(n=2 m)$ of two spheres in totally geodesic $\tilde{M}^{n+1}(\tilde{c})$ in $\tilde{M}^{n+2}(\tilde{c})$ or the product $S^{m}\left(\frac{1}{\sqrt{3 \tilde{c}}}\right) \times S^{m}\left(\frac{1}{\sqrt{3 \tilde{c}}}\right) \times S^{m}\left(\frac{1}{\sqrt{3 \tilde{c}}}\right)(n=3 m)$ of three spheres in $\tilde{M}^{n+2}(\tilde{c})$.

In this paper we would like to prove the following:

THEOREM 1. Let $M^{n}$ be an $n(>2)$-dimensional submanifold in $\tilde{M}^{n+p}(\tilde{c})$ with the parallel Ricci tensor. If the mean curvature normal $H$ is parallel and the normal connection of $M$ is trivial, then the second fundamental form of $M$ is parallel and $M$ is locally isometric to the product $M^{n_{1}} \times \cdots \times M^{n_{l}}, 1 \leqq l \leqq p+1$, where each $M^{n_{i}}$ is an $n_{i}$-dimensional sphere of some radius contained in some

Received November 27, 1987 
Euclidean space $N^{n_{i+1}}$ of dimension $n_{i}+1, N^{n_{i+1}} \perp N^{n^{+1}}$ for $i \neq j$, except possibly one of the $M^{n_{i}}$ is a Euclidean space $N^{n_{i}}$ (this can only occur if $\tilde{c} \leqq 0$ ) or hyperbolic space $H^{n_{i}}(\bar{c})$ with some negative curvature $\bar{c}$ (this can only occur if $\tilde{c}<0$ ).

Corollary 2. Let $M^{n}$ be an Einstein submanifold in $\tilde{M}^{n+2}(\tilde{c})$. If the mean curvature normal $H$ is parallel, then the second fundamental form of $M$ is parallel.

Remark 1. Let $f: M \rightarrow R^{3}$ be a surface of constant curvature $c \neq 0$, which is not contained in a sphere (See [1], p. 432). Embedd $R^{3}$ into $R^{4}$ and let $u$ be a unit vector orthogonal to $R^{3}$. Then $\tilde{f}: M \times R \rightarrow R^{4}$ which defined by $\tilde{f}(x, t)$ $=f(x)+t u$ gives an example of hypersurfaces in $R^{4}$ with parallel Ricci tensor, of which the second fundamental tensor is not parallel. By this example, the assumption on the mean curvature vector in Theorem 1 is necessary. Also, the assumption on the normal connection in Theorem 1 is not necessary if $p=2$ and $H \neq 0$ (See [2], Lemma 7).

Remark 2. In $S^{5}(1)$ the product $S^{1}\left(\frac{1}{\sqrt{3}}\right) \times S^{1}\left(\frac{1}{\sqrt{3}}\right) \times S^{1}\left(\frac{1}{\sqrt{3}}\right)$ of three spheres is a minimal Einstein submanifold. In $S^{6}(1)$ the product $S^{2}\left(\frac{1}{\sqrt{2}}\right)$ $\times S^{1}\left(\frac{1}{2}\right) \times S^{1}\left(\frac{1}{2}\right)$ of three spheres is a minimal submanifold with the parallel Ricci tensor. In $S^{7}(1)$ the product $S^{2}\left(\frac{\sqrt{2}}{\sqrt{5}}\right) \times S^{2}\left(\frac{\sqrt{2}}{\sqrt{5}}\right) \times S^{1}\left(\frac{1}{\sqrt{5}}\right)$ of three spheres and the product $S^{1}\left(\frac{1}{2}\right) \times S^{1}\left(\frac{1}{2}\right) \times S^{1}\left(\frac{1}{2}\right) \times S^{1}\left(\frac{1}{2}\right)$ of four spheres are minimal submanifolds with the parallel Ricci tensor. And, these normal connections are trivial.........(See [7]).

Finally, we would like to thank the referee for valuable comments.

\section{Submanifolds.}

Let $f$ be an isometric immersion of a connected Riemannian $n$-manifold $M^{n}$ into a real space form $\tilde{M}^{n+p}(\tilde{c})$ of constant curvature $\tilde{c}$. For all local formulas we may consider $f$ as an imbedding and thus identify $x \in M$ with $f(x) \in \tilde{M}$. The tangent space $T_{x} M$ is identified with a subspace of the tangent space $T_{x} \tilde{M}$. The normal space $T_{x}^{\perp}$ is the subspace of $T_{x} \tilde{M}$ consisting of all $X \in T_{x} \tilde{M}$ which are orthogonal to $T_{x} M$ with respect to the Riemannian metric g. Let $\nabla$ (resp. $\tilde{\nabla})$ denote the covariant differentiation in $M$ (resp. $\tilde{M}$ ), and $D$ the covariant differentiation in the normal bundle.

With each $\xi \in T_{\frac{1}{x}}^{+}$is associated a linear transformation of $T_{x} M$ in the following way. Extend $\xi$ to a normal vector field defined in a neighborhood of $x$ and define $-A_{\xi} X$ to be the tangential component of $\tilde{\nabla}_{X} \xi$ for $X \in T_{x} M$. $A_{\xi} X$ depends only on $\xi$ at $x$ and $X$. Given an orthonormal basis $\xi_{1}, \cdots, \xi_{p}$ of $T_{x}^{\perp}$ we write $A_{\alpha}=A_{\xi_{\alpha}}$ and call the $A_{\alpha}$ 's the second fundamental forms associated with $\xi_{1}, \cdots, \xi_{p}$. If $\xi_{1}, \cdots, \xi_{p}$ are now orthonormal normal vector fields in a 
neighborhood $U$ of $x$, they determine normal connection forms $s_{\alpha \beta}$ in $U$ by

$$
D_{x} \xi_{\alpha}=\sum_{\beta} s_{\alpha \beta}(X) \xi_{\beta}, \quad s_{\alpha \beta}+s_{\beta \alpha}=0
$$

for $X \in T_{x} M$. Let $X$ and $Y$ be tangent to $M$ and $\xi_{1}, \cdots, \xi_{p}$ orthonormal normal vector fields. Then we have the following relationships (in this section Greek indices run from 1 to $p$ ) [3]:

$$
\begin{aligned}
& \tilde{\nabla}_{X} Y=\nabla_{X} Y+\sigma(X, Y), \\
& \sigma(X, Y)=\sum_{\alpha} g\left(A_{\alpha} X, Y\right) \xi_{\alpha}, \quad g\left(A_{\alpha} X, Y\right)=g\left(A_{\alpha} Y, X\right), \\
& \left(\nabla_{X} A_{\alpha}\right) Y-\sum_{\beta} s_{\alpha \beta}(X) A_{\beta} Y=\left(\nabla_{Y} A_{\alpha}\right) X-\sum_{\beta} s_{\alpha \beta}(Y) A_{\beta} X
\end{aligned}
$$$$
R^{N}(X, Y) \xi_{\alpha}=\sum_{\beta} g\left(\left[A_{\alpha}, A_{\beta}\right] X, Y\right) \xi_{\beta},
$$

Codazzi equation,

$$
R i c=(n-1) \tilde{c} I+\sum_{\alpha}\left(\operatorname{trace} A_{\alpha}\right) A_{\alpha}-\sum_{\alpha} A_{\alpha}^{2},
$$

where $\sigma$ is also called the second fundamental form of $f$, and $R^{N}, R i c$ and $I$ denote the curvature tensor with respect to $D$, the Ricci tensor for $M$ and the identity transformation of $T_{x} M$, respectively.

The mean curvature normal $H$ is defined by

$$
H=\sum_{\alpha}\left(\operatorname{trace} A_{\alpha}\right) \xi_{\alpha},
$$

where the right side is independent of our choice of the orthonormal basis for $T \frac{1}{x}$. An immersion is said to be minimal if its mean curvature normal vanishes identically, i. e., if trace $A_{\alpha}=0$ for all $\alpha$.

\section{Proofs of Theorem 1 and Corollary 2.}

Let $f$ be an isometric immersion of $M^{n}$ into $\tilde{M}^{n+p}(\tilde{c})$ with the assumption of Theorem 1. From the results of [2], [6] and [7] we have only to prove that the second fundamental form of $M$ is parallel.

If $p=1$, then the theorem follows from Proposition 5 of Ryan [5].

We may assume that $p \geqq 2$. If $H \neq 0$ at $x$, then we can choose an orthonormal normal vector fields $\xi_{1}, \cdots, \xi_{p}$ defined in a neiborhood $U$ of $x$ such that $\xi_{1}=\frac{H}{|H|}$. Then on $U$ we have

$$
\text { trace } A_{1}=\text { constant and trace } A_{\beta}=0,2 \leqq \beta \leqq p .
$$

If $M$ is minimal, then as we of course have for any $\alpha$

$$
\text { trace } A_{\alpha}=0 \text {, }
$$

we may assume that (2.1) holds on $M$. Now since the normal connection is trivial, by continuity it is sufficient to prove that $\nabla A_{\alpha}=0$. In terms of (1.4) 
we have

$$
R i c=(n-1) \tilde{c} I+\sum_{\alpha}\left(\operatorname{trace} A_{\alpha}\right) A_{\alpha}-\sum_{\alpha} A_{\alpha}^{2}
$$

Then from (2.1) we have

$$
\sum_{\alpha}\left(\nabla A_{\alpha}\right) A_{\alpha}+\sum_{\alpha} A_{\alpha}\left(\nabla A_{\alpha}\right)-\left(\operatorname{trace} A_{1}\right) \nabla A_{1}=0 .
$$

On the other hand, from the triviality of the normal connection, i. e., $A_{\beta} A_{\gamma} \equiv$ $A_{\gamma} A_{\beta}$ for $1 \leqq \beta, \gamma \leqq p$ we have

$$
\left(\nabla A_{\beta}\right) A_{\gamma}+A_{\beta}\left(\nabla A_{\gamma}\right)-\left(\nabla A_{\gamma}\right) A_{\beta}-A_{\gamma}\left(\nabla A_{\beta}\right)=0 .
$$

Since $A_{\alpha}$ 's are also simultaneously parallelizable, we may consider $\lambda_{i}: T_{x}^{\perp} \rightarrow R$ so that

$$
A_{\alpha}=\left[\begin{array}{ccc}
\lambda_{1}\left(\xi_{\alpha}\right) & & 0 \\
0 & \ddots & \lambda_{n}\left(\xi_{\alpha}\right)
\end{array}\right] .
$$

Moreover we can take an orthonormal frame $\left\{e_{1}, \cdots, e_{n}\right\}$ of $M$ such that $A_{\alpha} e_{i}=$ $\lambda_{i}\left(\xi_{\alpha}\right) e_{i}$. Theorem 1 is trivial when $M^{n}$ is totally umblic. So we may assume that for some $\beta, A_{\beta} \neq \rho I$. Therefore we get $i \neq j$ such that $\lambda_{i}\left(\xi_{\beta}\right) \neq \lambda_{j}\left(\xi_{\beta}\right)$. For simplicity, put $\lambda=\lambda_{i}$ and $\mu=\lambda_{j}$. Now, put $A_{\beta} X=\lambda\left(\xi_{\beta}\right) X, A_{\gamma} X=\lambda\left(\xi_{\gamma}\right) X, A_{\beta} Y=$ $\mu\left(\xi_{\beta}\right) Y$ and $A_{\gamma} Y=\mu\left(\xi_{\gamma}\right) Y, \beta \neq \gamma$. Then from (2.2) we have

$$
\begin{aligned}
& \sum_{\alpha} \lambda\left(\xi_{\alpha}\right)\left(\nabla_{Y} A_{\alpha}\right) X+\sum_{\alpha} A_{\alpha}\left(\nabla_{Y} A_{\alpha}\right) X-\left(\operatorname{trace} A_{1}\right)\left(\nabla_{Y} A_{1}\right) X=0, \\
& \sum_{\alpha} \mu\left(\xi_{\alpha}\right)\left(\nabla_{X} A_{\alpha}\right) Y+\sum_{\alpha} A_{\alpha}\left(\nabla_{X} A_{\alpha}\right) Y-\left(\operatorname{trace} A_{1}\right)\left(\nabla_{X} A_{1}\right) Y=0 .
\end{aligned}
$$

Similarly, from (2.3) we have

$$
\begin{aligned}
& \lambda\left(\xi_{\gamma}\right)\left(\nabla_{Y} A_{\beta}\right) X+A_{\beta}\left(\nabla_{Y} A_{\gamma}\right) X-\lambda\left(\xi_{\beta}\right)\left(\nabla_{Y} A_{\gamma}\right) X-A_{\gamma}\left(\nabla_{Y} A_{\beta}\right) X=0, \\
& \mu\left(\xi_{\gamma}\right)\left(\nabla_{X} A_{\beta}\right) Y+A_{\beta}\left(\nabla_{X} A_{\gamma}\right) Y-\mu\left(\xi_{\beta}\right)\left(\nabla_{X} A_{\gamma}\right) Y-A_{\gamma}\left(\nabla_{X} A_{\beta}\right) Y=0 .
\end{aligned}
$$

Subtracting (2.5) from (2.4), using Codazzi equations (1.2), we have

$$
\sum_{\alpha}\left(\lambda\left(\xi_{\alpha}\right)-\mu\left(\xi_{\alpha}\right)\right)\left(\nabla_{X} A_{\alpha}\right) Y=0 .
$$

Similarly, from (2.6) and (2.7) we have

$$
\left(\lambda\left(\xi_{\gamma}\right)-\mu\left(\xi_{\gamma}\right)\right)\left(\nabla_{X} A_{\beta}\right) Y-\left(\lambda\left(\xi_{\beta}\right)-\mu\left(\xi_{\beta}\right)\right)\left(\nabla_{X} A_{\gamma}\right) Y=0 .
$$

Since $\lambda\left(\xi_{\beta}\right) \neq \mu\left(\xi_{\beta}\right)$ by the assumption, we get

$$
\left(\nabla_{X} A_{\gamma}\right) Y=\frac{\lambda\left(\xi_{\gamma}\right)-\mu\left(\xi_{\gamma}\right)}{\lambda\left(\xi_{\beta}\right)-\mu\left(\xi_{\beta}\right)}\left(\nabla_{X} A_{\beta}\right) Y
$$

for any $\gamma$. Substituting this into (2.8), we obtain 


$$
\left(\nabla_{X} A_{\beta}\right) Y=0 \text {. }
$$

From (2.9), it follows $\left(\nabla_{X} A_{\gamma}\right) Y=0$ for all $\gamma$.

This proves Theorem 1 .

Next, we prove Corollary 2. Let $M^{n}$ be an Einstein submanifold in $\tilde{M}^{n+2}(\tilde{c})$ with the parallel mean curvature normal $H$.

If $H \neq 0$ at $x$, then as in the above we choose an orthonormal normal vector fields $\xi_{1}, \xi_{2}$ defined in a neighborhood $U$ of $x$ such that $\xi_{1}=\frac{H}{|H|}$. Now $D H=0$ implies $D \xi_{1}=0$ and hence $s_{12}=0$ in $U$. This implies $U$ satisfies the assumption of Theorem 1. Hence the second fundamental form of $M$ is parallel in $U$. If there exists a neighborhood $V$ which satisfies $H \equiv 0$, then $V$ holds the assumption of Theorem. Hence the second fundamental form of $M$ is parallel in $V$. By continuity we obtain that the second fundamental form of $M$ is parallel.

This proves Corollary 2 .

\section{REFERENCES}

[1] M. Berger and B. Gostiaux, Géométrie différentielle: variétés, courbes et surfaces, Press Universitaires, de France, 1987.

[2] J. ERBACHER, Isometric immersions of constant mean curvature and triviality of the normal connection, Nagoya Math. J. 45 (1971), 139-165.

[3] Y. Matsuyama, On minimal Einstein submanifolds with codimension two, to appear.

[4] Y. Matsuyama, Minimal Einstein submanifolds, to appear.

[5] P. J. RYAN, Hypersurfaces with parallel Ricci tensor, Osaka J. Math. 8 (1971), 251-259.

[6] M. TAKeUChI, Parallel submanifolds of space forms, Manifolds and Lie groups (Papers in honor of Y. Matsushima) edited by J. Hano, etc. Birkhäuser, 1981.

[7] K. YANO AND S. IshiHARA, Submanifolds with parallel mean curvature vector, J. Differential Geom. 6 (1971), 95-118.

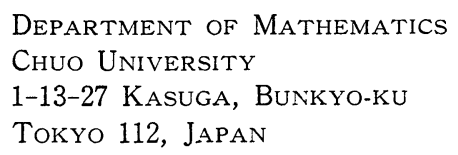

